\title{
Irreversibilität und Quantentheorie
}

\author{
Von Hermann Kümael \\ Aus dem Institut für theoretische Physik der Freien Universität Berlin \\ (Z. Naturforschg. 11 a, 15-20 [1956] ; eingegangen am 14. September 1955)
}

\begin{abstract}
Vom Verfasser ${ }^{1,2}$ wurden die Phasenraumdichte und ihre als Differenzengleichung erscheinende klassische Bewegungsgleichung auf quantentheoretischer Grundlage abgeleitet. In dieser Arbeit wird das Erreichen des Gleichgewichtes als Vorgang im $(p-q)$-Raum untersucht. Das statistische Grundgesetz (Bewegungsgleichungen der Dichte, ausgedrückt durch Übergangswahrscheinlichkeiten $W_{J J^{\prime}}$ ) ist kein spezifisch quantenmechanisches Gesetz. Erst durch eine „Vergröberung“ der $W_{J J^{\prime}}$ und die dann ablesbaren Symmetrierelationen liefert das statistische Grundgesetz quantitativ den Übergang in das Gleichgewicht und die irreversible Thermodynamik in der O n s a g e r schen Form.
\end{abstract}

\section{$\S 1$. Einleitung}

In letzter Zeit ist das Verständnis der quantentheoretischen Grundlagen der statistischen Mechanik hinsichtlich folgender Punkte erheblich gefördert worden:

1. "Geometrische Eigenschaften" des Vielteilchensystems: Wir meinen damit die aus geometrischen Bedingungen folgenden Eigenschaften der Eigenwerte und Eigenfunktionen. Hier muß man - wegen der Hoffnungslosigkeit jeglicher exakten Rechnung - gewisse Forderungen stellen: Die Eigenwerte müssen sehr dicht liegen und hinreichend ungeordnet sein (s. Ludwig ${ }^{3}, \mathrm{~K} \ddot{\mathrm{um}}$ $\mathrm{mel}^{1}$ ). Die Eigenfunktionen müssen die Form $A_{n} \exp \left[i W_{n} / \hbar\right]$ mit langsam veränderlichen $A_{n}$ und $\partial / \partial x \cdot W_{n}$ haben (jedenfalls solange die Ortskoordinaten zweier Teilchen sich nicht sehr nahe kommen, s. K I, Kap. 2).

2. Definition der makroskopischen Observablen: Als solche kommen in der Energiedarstellung nur beinahe diagonale Operatoren in Frage ( $\mathrm{Ludwig}^{3}$, v a n $\mathrm{K}$ a mpen ${ }^{4}$, K I). Dies deshalb, weil sie praktisch vertauschbar sein müssen und nur langsam von der Zeit abhängen dürfen (jede makroskopische Messung impliziert eine Zeitmittelung).

3. Herstellung makroskopischer Observablen: Es lassen sich alle notwendigen Observablen als Integrale über einen makroskopischen Bereich bilden (s. K I, 3.1). Die Integranden sind die Matrixelemente der quantentheoretischen

${ }^{1}$ H. K ü m m e l, Nuovo Cim. 1, 1057 [1955], im folgenden als $\mathrm{K} I$ zitiert.

${ }^{2}$ H. K ü m m e l, Nuovo Cim. 2, 877 [1955], im folgenden als $\mathrm{K}$ II zitiert.
Operatoren. Solche Integrale haben die Eigenschaft, beinahe diagonal zu sein. Die Mittelung über einen makroskopischen Bereich ist notwendig, weil die mikroskopischen lokalen Schwankungen makroskopisch nicht beobachtbar sind. Dann resultieren die klassischen Bewegungsgleichungen als Differenzengleichungen.

4. Anschluß an die klassische Statistik und ihre Methoden: Es läßt sich eine Phasendichte einführen, die die klassischen Bewegungsgleichungen erfüllt (s. K II). Dies ist richtig bei nicht zu tiefer Temperatur. Korrektionen hierzu sind aber ohne weiteres durchführbar (vgl. hierzu $\mathrm{Kümmel}{ }^{5}$ ). Die Bewegungsgleichungen sind jedoch wiederum Differenzengleichungen.

5. Erreichen des Gleichgewichts: Von Ludwig ${ }^{3}$ ist gezeigt, daß die makroskopischen Observablen im Zeitmittel durch ihre konstanten Diagonalelemente ersetzt werden können, daß diese also einem Gleichgewicht zustreben. Daraus folgt, daß die Phasenraumdichte im Mittel über die Energiefläche gleichmäßig verteilt ist (K II, 2.4). $\mathrm{V}$ a n Kampen ${ }^{4}$ hat (allerdings nur für einen Zustand) ohne Bezugnahme auf den klassischen $(p-q)$-Phasenraum das Erreichen des Gleichgewichts quantitativ nach der Gleichung

$$
\dot{\widetilde{\mho}}(J)=\sum_{J^{\prime}}\left(W_{J J^{\prime}} \tilde{\mathscr{J}}\left(J^{\prime}\right)-W_{J^{\prime} J} \widetilde{\mathscr{J}}(J)\right)
$$

und der Symmetrierelation

$$
W_{J J^{\prime}}=W_{-J^{\prime}-J}
$$

beschreiben können. (Zur Definition der Größen

${ }^{3}$ G. Lu dwig, Z. Phys. 135, 483 [1953] und: Die Grundlagen der Quantenmechanik, Berlin, Göttingen, Heidelberg, 1954.

${ }_{4}^{4}$ N. G. V a n K a m p e n, Physica 20, 603 [1954].

5 H. K ü m m e l, Z. Phys. 143, 219 [1955]. 
vgl. die Arbeit von van $\mathrm{Kampen}{ }^{4}$ bzw. unten $\S 2$ und $\S 3$; $-J$ bezeichnet eine der Stelle $J$ im Phasenraum in bestimmter Weise zugeordnete Stelle.)

In der folgenden Arbeit soll nun dieser Punkt 5 ergänzt und gegenüber den Methoden von van $\mathrm{K}$ a m pen ${ }^{4}$ erweitert werden: Die beiden statistischen Grundgleichungen (1) und (2) sollen als Gleichungen im Phasenraum formuliert werden. Ferner soll geklärt werden, wie die ursprünglich gegen Zeitumkehr invarianten Bewegungsgleichungen für $\mathfrak{F}$ in die Gln. (1) und (2) übergehen, die gerade die Irreversibilität liefern. Es wird sich zeigen, daß die klassischen Bewegungsgleichungen sich in der Form (1) schreiben lassen, daß aber klassisch eine Symmetrierelation (2) nicht gilt. Erst gewisse Vernachlässigungen in der Wellenfunktion (bzw. in Produkten von solchen) geben die Relation (2). Dann gilt also die Irreversibilität nur bis auf kleine Glieder, und zwar, wie sich zeigen wird, von der Größenordnung $(\Delta / \Delta x) \tilde{\mho} \Delta x$. Nur wenn ,infinitesimal“ benachbarte Stellen des Phasenraums als gleichberechtigt betrachtet werden, gilt der Ergodensatz. Das ist die bekannte Tatsache, daß die Phasenbahn jedem Punkt der Energiefläche „beliebig nahe kommt". Erst die Quantentheorie liefert also eine vollständige Beschreibung dieses Sachverhaltes, d.h. die quantitative Formulierung des Ergodensatzes.

Es ist klar, daß diese Erkenntnisse auch das Verständnis der Irreversibilität der Boltzmannschen Stoßgleichung fördern. Hierüber ist in einer anderen Arbeit berichtet worden ${ }^{5}$.

\section{$\S$ 2. Das statistische Grundgesetz}

In $\mathrm{K}$ II ist gezeigt, daß die Verteilungsfunktion $(l=1 \ldots N)$

Es ist leicht, $\widetilde{\mho}_{N}\left(\mathfrak{V}_{l}, \mathfrak{K}_{l}, t\right)$ durch $\widetilde{\mho}_{N}\left(\mathfrak{V}_{l}, \mathfrak{K}_{l}, \tau\right)$ auszudrücken. Dazu berechnen wir $w_{n m}$ für die Zeit $t=\tau$ aus Gl. (3) : Wir brauchen nämlich nur

$$
\begin{gathered}
w_{n m}=\int \mathrm{d} \mathfrak{r}_{l}^{\prime} \int \mathrm{d} \mathfrak{v}_{l}^{\prime} \int \overline{\mathrm{d}} \overline{\mathbf{r}}_{l}^{\prime} \exp \left[i / \hbar m \sum \mathfrak{v}_{l}^{\prime}{\overline{\mathbf{r}_{l}}}^{\prime}\right] \cdot \sum( \pm 1)^{P+P^{\prime}} \psi_{n}{ }^{* P}\left(\mathfrak{r}_{l}^{\prime}+\frac{1}{2} \overline{\mathfrak{r}_{l}^{\prime}}\right) \psi_{m}{ }^{P^{\prime}}\left(\mathfrak{r}_{l}^{\prime}-\frac{1}{2} \mathfrak{r}_{l}^{\prime}\right) \\
\cdot \widetilde{\mho}_{N}\left(\mathfrak{V}_{l}^{\prime}, \Re_{l}^{\prime}, \tau\right) \exp \left[-i \omega_{m n} \tau\right]
\end{gathered}
$$

zu setzen. Das prüfen wir leicht nach, indem wir (5) in (3) für $t=\tau$ einsetzen; wegen

$$
\sum_{n} \psi_{n}^{*}(\mathfrak{r}) \psi_{n}\left(\mathfrak{r}^{\prime}\right)=\delta\left(\mathfrak{r}-\mathfrak{r}^{\prime}\right) \quad \text { gibt das sofort } \quad \mathfrak{r}_{l}=\mathfrak{r}_{l}^{\prime}, \quad \overline{\mathfrak{r}}_{l}=\overline{\mathfrak{r}}_{l}^{\prime},
$$

${ }^{6}$ Außerdem ist fast immer $\mathfrak{F}_{N} \geq 0$; vgl. H. K ü m mel, Nuovo Cim., im Druck. (bei hinreichend hoher Temperatur) die klassische Bewegungsgleichung

$$
\begin{aligned}
\frac{\partial \mathfrak{F}_{N}\left(\mathfrak{B}_{l}, \mathfrak{R}_{l}, t\right)}{\partial t}+ & \sum_{i=1}^{N} \mathfrak{V}_{v}(i) \frac{\Delta \mathfrak{F}_{N}}{\Delta \mathfrak{R}_{v}(i)} \\
& -\frac{1}{m} \sum_{i=1}^{N} V_{\mathfrak{i}_{i}}\left(\mathfrak{K}_{l}\right) \frac{\Delta \mathfrak{F}_{N}}{\Delta \mathfrak{B}_{v}(i)}=0
\end{aligned}
$$

erfüllt. Wir haben dabei

$$
\underset{i}{\mathfrak{f}_{v}} \equiv \frac{\Delta f}{\Delta \mathfrak{R}_{v}^{(i)}} \equiv \frac{f\left(\mathfrak{R}_{v}^{(i)}+\Delta \mathfrak{R}_{v}^{(i)}\right)-f\left(\mathfrak{R}_{v}^{(i)}\right)}{\Delta \mathfrak{R}_{v}^{(i)}}
$$

gesetzt. Die Bedeutung als Verteilungsfunktion ergibt sich aus den Gleichungen für den Erwartungswert makroskopischer Observablen

$\operatorname{Erw}(A)=\int A\left(\mathfrak{K}_{l}, \mathfrak{V}_{l}, 0\right) \mathfrak{\mho}_{N}\left(\mathfrak{K}_{l}, \mathfrak{V}_{l}, t\right) \mathrm{d} \mathfrak{V}_{l} \mathrm{~d} \mathfrak{H}_{l}$ und

$$
\int \mathfrak{\mho}_{N}\left(\mathfrak{V}_{l}, \mathfrak{\Re}_{l}, t\right) \mathrm{d} \mathfrak{V}_{l} \mathrm{~d} \Re_{l}=1^{6} \quad \text { (vgl. K II). }
$$


so daß sich die Integrationen über $\mathfrak{r}_{l}^{\prime}$ und $\overline{\mathfrak{r}}_{l}^{\prime}$ sofort ausführen lassen:

$$
\begin{aligned}
\widetilde{\mho}_{N}\left(\mathfrak{V}_{l}, \mathfrak{K}_{l}, \tau\right)=\frac{1}{\Delta \tau^{N}}\left(\frac{m}{2 \pi \hbar}\right)^{3 N} \cdot \int_{\Delta \tau_{l}} \mathrm{~d}_{l} \mathrm{dr}_{l} \int \mathrm{d} \mathfrak{r}_{l} \int \mathrm{d} \mathfrak{v}_{l}^{\prime} \exp \left[-\frac{i}{\hbar} m \sum\left(\mathfrak{v}_{l}-\mathfrak{v}_{l}^{\prime}\right) \overline{\mathfrak{r}}_{l}\right] \widetilde{\mho}_{N}\left(\mathfrak{V}_{l}^{\prime}, \mathfrak{K}_{l}, \tau\right) & =\frac{1}{\Delta \tau^{N}} \int_{\Delta \tau_{l}} \mathrm{~d} \mathfrak{v}_{l} \mathrm{dr}_{l} \widetilde{\mho}_{N}\left(\mathfrak{V}_{l}, \mathfrak{\Re}_{l}, \tau\right), \quad \text { q. e. d. }
\end{aligned}
$$

Denken wir uns nun (5) für beliebiges $t$ in (3) eingesetzt, so steht dort:

mit

$$
\widetilde{\mho}_{N}\left(\mathfrak{V}_{l}, \mathfrak{R}_{l}, t\right)=\int \mathrm{d} \mathfrak{V}_{l}^{\prime} \mathrm{d} \mathfrak{\Re}_{l}^{\prime}\left[\mathfrak{V}_{l} \mathfrak{\Re}_{l} \mid \mathfrak{V}_{l}^{\prime} \mathfrak{K}_{l}^{\prime}\right]_{t-\tau} \widetilde{\mho}_{N}\left(\mathfrak{V}_{l}^{\prime}, \mathfrak{K}_{l}^{\prime}, \tau\right)
$$

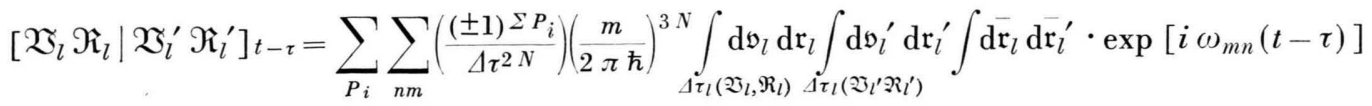

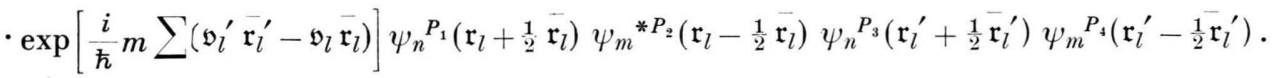

Um den Anschluß an die Terminologie und Methode von $\mathrm{v}$ a $\mathrm{K}$ a m pe ${ }^{4}$ herzustellen, setzen wir

$$
\left(\mathfrak{V}_{l}, \mathfrak{K}_{l}\right) \rightarrow J
$$

und haben dann

$$
\widetilde{\mho}_{N}(J, t)=\sum_{J^{\prime}}\left[J \mid J^{\prime}\right]_{t-\tau} \widetilde{\mho}_{N}\left(J^{\prime}, \tau\right) \text {. }
$$

Offensichtlich gilt

$$
\left[\mathfrak{V}_{l} \mathfrak{K}_{l} \mid \mathfrak{V}_{l}^{\prime} \mathfrak{K}_{l}^{\prime}\right]_{0}=\delta\left(\mathfrak{V}_{l}-\mathfrak{V}_{l}^{\prime}\right) \delta\left(\mathfrak{K}_{l}-\mathfrak{K}_{l}^{\prime}\right)
$$

bzw.

$$
\left[J \mid J^{\prime}\right]_{0}=\delta_{J J^{\prime}} .
$$

Wir können daher

$$
\begin{aligned}
& W\left(\mathfrak{V}_{l} \mathfrak{K}_{l} \mid \mathfrak{V}_{l}^{\prime} \mathfrak{K}_{l}^{\prime}\right) \\
= & \lim _{\Delta t \rightarrow 0} \frac{1}{\Delta t}\left\{\left[\mathfrak{V}_{l} \mathfrak{K}_{l} \mid \mathfrak{V}_{l}^{\prime} \mathfrak{K}_{l}^{\prime}\right]_{\Delta t}-\left[\mathfrak{V}_{l} \mathfrak{K}_{l} \mid \mathfrak{V}_{l}^{\prime} \mathfrak{K}_{l}^{\prime}\right]_{0}\right\}
\end{aligned}
$$

bzw.

$$
W_{J J^{\prime}}=\lim _{\Delta t \rightarrow 0} \frac{1}{\Delta t}\left\{\left[\boldsymbol{J} \mid \boldsymbol{J}^{\prime}\right]_{\Delta t}-\delta_{J J^{\prime}}\right\}
$$

als zeitunabhängige Größen einführen. Dann gilt nach $(6 a)$

$$
\frac{\partial \widetilde{F}_{N}(J, t)}{\partial t}=\sum_{J^{\prime}} W_{J J^{\prime}} \widetilde{\mho}_{N}\left(J^{\prime}, t\right) .
$$

Da schließlich

$$
\sum_{J} \widetilde{\mho}_{N}(J, t)=1
$$

normiert werden kann, folgt aus (10)

$$
\sum_{J} W_{J J^{\prime}}=0
$$

d. h. es wird das erste statistische Grundgesetz:

$$
\frac{\partial \widetilde{F}_{N}(J, t)}{\partial t}=\sum_{J^{\prime} \neq J}\left\{W_{J J^{\prime}} \widetilde{\mho}_{N}\left(J^{\prime}, t\right)-W_{J^{\prime} J} \widetilde{\mho}_{N}(J, t)\right\} .
$$

Es sei speziell

$$
\widetilde{\mho}_{N}\left(J_{0}, t\right)=1, \quad \widetilde{\mho}_{N}(J, t)=0 \text { für } J \neq J_{0} .
$$

Dann muß „fast immer“ (vgl. Anm. ${ }^{6}$ )

$$
\dot{\widetilde{\mho}}_{N}(J, t) \geqq 0, \quad \dot{\widetilde{\mho}}_{N}\left(J_{0}, t\right) \leqq 0
$$

sein, also

$$
\dot{\widetilde{\mho}}_{N}(J, t)=W_{J J_{0}} \widetilde{\mho}_{N}\left(J_{0}, t\right)=W_{J J_{0}} .
$$

Daraus folgt

$$
W_{J J_{0}} \geqq 0 \quad \text { und } \quad W_{J J} \leqq 0 .
$$

Da $W_{J J^{\prime}}$ von $\tilde{\mho}_{N}$ und $t$ unabhängig ist, gilt (14) allgemein.

Wir haben also (im allgemeinen) zwei Bewegungsgleichungen für $\widetilde{\mho}_{N}$, nämlich (4) und (13). Beide sind (für nicht zu tiefe Temperaturen) äquivalent. Insbesondere leitet man leicht eine Form für $W_{J J^{\prime}}$ ab, die direkt Gl. (4) entspricht (und die benachbarte Phasenpunkte miteinander kombiniert). Es macht auch keine Schwierigkeiten, aus (13) die Gl. (4) abzuleiten, indem man benutzt, da $3 W_{J J^{\prime}}$ fast dasselbe ist wie $\left[J \mid J^{\prime}\right]$; man muß im letzteren Ausdruck lediglich

$\exp \left[i \omega_{m n}(t-\tau)\right] \quad$ durch $\quad i \omega_{m n}=\frac{i}{\hbar}\left(\varepsilon_{m}-\varepsilon_{n}\right)$

ersetzen und

$$
\frac{\hbar}{i} \dot{\psi}_{n}=\varepsilon_{n} \psi_{n}=\left(-\frac{\hbar^{2}}{2 m} \sum_{i}^{N} \Delta_{i}+\sum_{i<k}^{N} V_{i k}\right) \psi_{n}
$$

beachten. Wir wollen dies hier nicht im einzelnen durchrechnen.

Wesentlich ist, daß (13) mit dem durch (9) definierten $W_{J J^{\prime}}$ invariant gegen Zeitumkehr ist, weil (4) es ist. Das erste statistische Grundgesetz allein liefert also nicht die Irreversibilität. 


\section{§ 3. „Vergröberung" des statistischen Grundgesetzes}

Nach den eben angestellten Überlegungen wollen wir die Gleichungen „vergröbern“, denn es ist zu erwarten, daß erst eine hinreichend ungenaue Gleichung, bei der benachbarte Punkte im Phasenraum gleichberechtigt sind, eine Auszeichnung der Zeitrichtung zur Folge hat. Dazu betrachten wir die Gl. (7) für $\left[J \mid J^{\prime}\right]_{t-\tau}$. Wir benutzen jetzt die im $\S 1$ erwähnten Voraussetzungen über die Wellenfunktionen: Sie sollen die Form

$$
A_{n} \exp \left[i W_{n} / \hbar\right]
$$

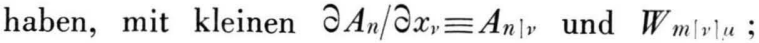
während diese Größen als von erster Ordnung klein bisher (s. K I und K II) immer mitberücksichtigt wurden, sollen sie jetzt unterschlagen werden. Die räumliche Änderung von $A_{n}$ und $W_{m i v}$ im makroskopischen Intervall $\Delta \mathfrak{i}$ wird also vernachlässigt.

Entwickeln wir

mit $\left\{\begin{array}{l}W_{n m}=W_{n}-W_{m}, l=1 \ldots N, v=1 \ldots 3 \\ W_{n+m}=W_{n}+W_{m}\end{array}\right.$

$W_{n}\left(\mathfrak{r}_{l}-\frac{1}{2} \overline{\mathfrak{r}}_{l}\right)-W_{m}\left(\mathfrak{r}_{l}+\frac{1}{2} \overline{\mathfrak{r}}_{l}\right) \approx W_{n m}(\mathfrak{r})-W_{m+n \mid v} \overline{\mathfrak{r}}_{l}$

(was man machen kann, da die Integrale über $\mathfrak{b}_{l}$ bzw. $\mathfrak{v}_{l}^{\prime}$ dafür sorgen, daß $\overline{\mathfrak{r}}_{l}, \overline{\mathfrak{r}}_{l}^{\prime}$ klein bleibt), dann erhält man statt $(7)$

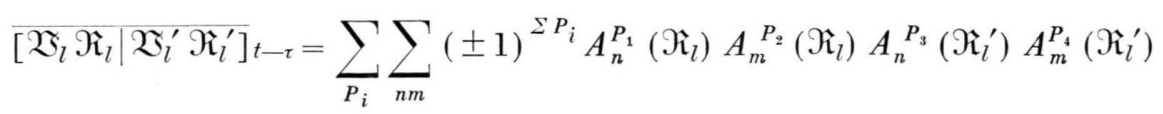

$$
\begin{aligned}
& \cdot \frac{1}{(\Delta \mathfrak{R})^{6 N}} \int_{\Delta V\left(\Re_{l}\right)} \mathrm{dr}_{l} \int_{\Delta V\left(\Re l^{\prime}\right)} \mathrm{dr}_{l}^{\prime} \exp \left[\frac{i}{\hbar}\left\{W_{n m}^{P_{1} P_{2}}(\mathfrak{r})-W_{n m}^{P_{3} P_{4}}\left(\mathfrak{r}^{\prime}\right)\right\}\right] \exp \left[i \omega_{m n}(t-\tau)\right] \\
& \cdot \bar{\delta}\left(m \mathfrak{V}_{v}^{(l)}-\frac{1}{2} W_{n+m, v}^{P_{1} P_{2}}\left(\Re_{l}\right)\right) \bar{\delta}\left(m \mathfrak{V}_{v}^{(l) \prime}-\frac{1}{2} W_{n+m, v}^{P_{3} P_{4}}\left(\Re_{l}^{\prime}\right)\right)
\end{aligned}
$$

mit „breiten“ $\delta$-Funktionen $\left(\Delta \mathfrak{V}_{l} \geqq \frac{\hbar}{m} \frac{1}{\Delta \mathfrak{R}_{l}}\right)$.

Diese Matrix ist bis auf Glieder erster Ordnung mit der Matrix $\left[\mathfrak{V}_{l} \mathfrak{K}_{l} \mid \mathfrak{V}_{l}^{\prime} \mathfrak{K}_{l}^{\prime}\right]_{t-\tau}$ identisch. Mit

$$
\bar{W}_{J J^{\prime}}=\lim _{\Delta t \rightarrow 0} \frac{1}{\Delta t}\left\{\left[\bar{J}^{\prime} \mid J^{\prime}\right]_{\Delta t}-\bar{\delta}_{J J^{\prime}}\right\}
$$

können wir dann das statistische Grundgesetz in die Form

$$
\dot{\overrightarrow{\widetilde{F}}}_{N}(J, t)=\sum_{J^{\prime}}\left\{\bar{W}_{J J^{\prime}} \overline{\widetilde{\mho}}_{N}\left(J^{\prime}, t\right)-\bar{W}_{J^{\prime} J} \widetilde{\widetilde{F}}(J, t)\right\}
$$

bringen, eine Gleichung, die $\widetilde{\mho}_{N}$ nur in „nullter Ordnung" bestimmt, was wir durch Überstreichen angedeutet haben.

Wir wollen noch überlegen, was unsere Vernachlässigungen bedeuten. Dazu betrachten wir z. B. $\Delta \mathfrak{F}_{N} / \Delta \mathfrak{\Re}_{v}$; wir haben Integrale

$$
\frac{1}{(\Delta \mathfrak{R})^{3}} \int_{\Delta V(\mathfrak{R})} \psi_{n}^{*}(\mathfrak{r}) \psi_{m}(\mathfrak{r}) \mathrm{d} \mathfrak{r}
$$

makroskopisch zu differenzieren (vgl. K I, 2.3) :

$$
\begin{aligned}
& \frac{\Delta}{\Delta \mathfrak{R}_{v}} \frac{1}{(\Delta \mathfrak{R})^{3}} \int_{\Delta V(\mathfrak{R})} \psi_{n}{ }^{*} \psi_{m} \mathrm{dr} \\
& =\left(A_{n}(\Re) A_{m}(\Re)\right)_{v} \cdot \frac{1}{(\Delta \mathfrak{R})^{3}} \int \exp \left[\frac{i}{\hbar} W_{n m}\right] \mathrm{dr} \\
& \quad+\frac{i}{\hbar} W_{n m \mid v}(\Re) \frac{1}{(\Delta \mathfrak{R})^{3}} \int_{\Delta V} \psi_{n}^{*} \psi_{m} \mathrm{dr} .
\end{aligned}
$$

Das erste Glied der rechten Seite lassen wir nach dem oben Gesagten als klein von erster Ordnung weg. Auch das letzte Glied wird effektiv klein, um so kleiner, je geringer die Abweichungen vom Gleichgewicht sind. Denn bei der Bildung von Erwartungswerten mit makroskopischen Observablen geben nur fast-diagonale Elemente Beiträge (vgl. K II, 2.4). Wir brauchen also nur fast-diagonale $\omega_{n m}$ in (3) in Betracht zu ziehen. Wir dürfen allerdings dies letzte Glied nicht ohne weiteres weglassen $\left(W_{n m \text { Iv }}\right.$ bestimmt entscheidend das Verhalten von $\bar{W}_{J J^{\prime}}$, wie wir gleich noch sehen werden). Daher können wir sagen, daß $\Delta \widetilde{\mho}_{N} / \Delta \Re$ zum Teil vernachlässigt ist; mit anderen Worten: wegen

$$
\widetilde{\mho}_{N}\left(\Re+\Delta \Re_{v}\right)=\widetilde{J}_{N}(\mathfrak{K})+\frac{\Delta}{\Delta \mathfrak{R}_{v}} \widetilde{\mho}_{N}(\mathfrak{K}) \Delta \mathfrak{H}_{v}
$$

ist $\widetilde{\mho}_{N}\left(\Re+\Delta \Re_{v}\right) \approx \widetilde{\mho}_{N}(\Re)$ gesetzt. D. h. „infinitesimal" benachbarte Punkte werden als gleiche Punkte angesehen.

\section{§ 4. Symmetrieeigenschaft und Irreversibilität}

An der oben gewonnenen ungenauen Form der Übergangswahrscheinlichkeiten läßt sich leicht die Symmetrierelation 


$$
\bar{W}\left(\mathfrak{V}_{l} \mathfrak{K}_{l} \mid \mathfrak{V}_{l}^{\prime} \mathfrak{K}_{l}^{\prime}\right)=\bar{W}\left(-\mathfrak{V}_{l}^{\prime} \mathfrak{K}_{l}^{\prime} \mid-\mathfrak{V}_{l} \mathfrak{K}_{l}\right) \quad \text { bzw. } \quad \bar{W}_{J J^{\prime}}=\bar{W}_{-J^{\prime}-J}
$$

beweisen, wenn wir $J \rightarrow\left(\mathfrak{V}_{l} \mathfrak{H}_{l}\right), \quad-J \rightarrow\left(-\mathfrak{V}_{l} \mathfrak{H}_{l}\right)$ setzen. Man erkennt das aus Gl. (16), die wir mit (15)

$$
\begin{aligned}
& \bar{W}\left(\mathfrak{V}_{l} \mathfrak{K}_{l} \mid \mathfrak{V}_{l}^{\prime} \Re_{l}^{\prime}\right)=\sum_{n m} i \omega_{m n} \sum_{P_{i}}( \pm 1)^{\Sigma P_{i}} \cdot A_{n}^{P_{1}}\left(\Re_{l}\right) A_{m}^{P_{2}}\left(\Re_{l}\right) A_{m}^{P_{3}}\left(\Re_{l}^{\prime}\right) A_{n}^{P_{4}}\left(\Re_{l}^{\prime}\right)
\end{aligned}
$$

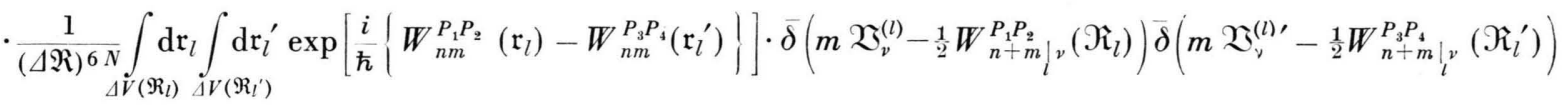

schreiben können. Wegen $\omega_{m n}=-\omega_{n m}$ wird daraus

$$
\begin{aligned}
& \bar{W}\left(\mathfrak{V}_{l} \mathfrak{K}_{l} \mid \mathfrak{V}_{l}^{\prime} \mathfrak{\Re}_{l}^{\prime}\right)=2 \sum_{m>n} \omega_{n m} \sum_{P_{i}}( \pm 1)^{\Sigma P_{i}} \cdot A_{n}^{P_{1}}\left(\Re_{l}\right) A_{m}^{P_{9}}\left(\Re_{l}\right) A_{n}^{P_{3}}\left(\Re_{l}^{\prime}\right) A_{m}^{P_{4}}\left(\Re_{l}^{\prime}\right)
\end{aligned}
$$

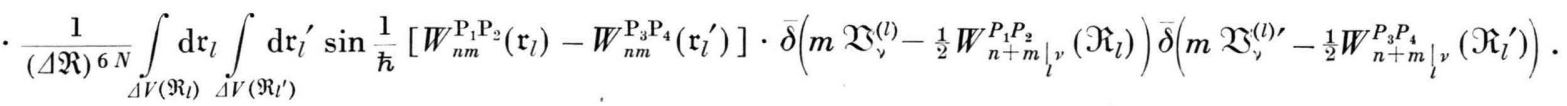

Nun greifen wir auf die Schrödinger-Gleichungen

$$
\left.\begin{array}{l}
2 m\left(\varepsilon_{n}-V\right)=\sum_{i=1}^{N}\left(\begin{array}{c}
\left.W_{n \mid v}\right)^{2}, \\
i,
\end{array}\right. \\
\sum_{i=1}^{N} W_{\substack{n|v| v \\
i \quad}}+2 A_{n} \sum_{i=1}^{N} A_{n \mid v}, W_{n \mid v}=0 \\
i
\end{array}\right\}
$$

zurück. Aus diesen liest man leicht ab, daß

$$
W_{n}\left(\mathfrak{r}_{l}\right) \approx \alpha_{n}+\sum_{l} W_{n \mid v}\left(\mathfrak{r}_{l}\right) \mathfrak{r}_{v}(l)
$$

gilt mit überall konstantem Phasenwinkel $\alpha_{n}$. (In unserer Rechnung ist auch $W_{n i v}$ konstant.) Man kann auch sagen: Die Ortsabhängigkeit läßt sich in $A_{n}$ und $W_{n \mid v}$ hineinstecken, solange der HamiltonOperator „reell“ ist, also keine Impulse linear enthält. Dann ist wegen des Integrals über $\mathfrak{r}^{\prime}$ bzw. $\mathfrak{r}$ in $(20) W_{n \mid v} \approx W_{m \mid v}$. (Nur solche Wellenfunktionen geben merkliche Beiträge.) $\mathrm{Zu}$ jedem $W_{n 1 v}>0$ gibt es ein gleichberechtigtes gleich großes $W_{n \mid v}<0$. Wir können also in den $\bar{\partial}$-Funktionen $\mathfrak{V}$ durch $-\mathfrak{V}$ und $\mathfrak{W}^{\prime}$ durch - $\mathfrak{J}^{\prime}$ ersetzen, wenn wir nur im $\sin W_{n m}$ durch $-W_{n m}$ ersetzen. Der so aus (20) erhaltene Ausdruck ist dann aber nichts anderes als

$$
\bar{W}\left(-\mathfrak{V}_{l}^{\prime}, \mathfrak{\Re}_{l}^{\prime} \mid-\mathfrak{V}_{l}, \mathfrak{K}_{l}\right), \quad \text { q. e. d. }
$$

Damit ist aber die Irreversibilität gesichert. Es gilt nämlich nach der Symmetrierelation (18) und Gl. (12) :

$$
\sum_{J^{\prime}} \bar{W}_{J J^{\prime}}=\sum_{J^{\prime}} \bar{W}_{J^{\prime}-J}=0 .
$$

Zusammen mit dem statistischen Grundgesetz (17) liefert (23) dann, wie $\mathrm{Thomsen}{ }^{7,8}$ gezeigt hat,

\footnotetext{
7 J. S. Th o m se n, Phys. Rev. 91, 1263 [1953].
}

das Ergebnis, daß $\overline{\widetilde{F}}\left(\mathfrak{V}_{l}, \Re_{l}, t\right)$ der Gleichverteilung auf der Energieschale zustrebt.

An dieser Stelle müßte man noch beweisen, daß $\bar{W}_{J J^{\prime}}$ nur solche Punkte des Phasenraumes miteinander verbindet, die zu derselben Energiefläche gehören. Da aber $W_{J J^{\prime}}$ dies sicher tut, kann man sagen, daß auch $W_{J J^{\prime}}$ näherungsweise (mit der hier notwendigen geringen Genauigkeit) diese Forderung erfüllt. Benutzt man die in K II durchgeführte Ableitung der klassischen Bewegungsgleichung und läßt man dort alle Glieder erster Ordnung fort, so erhält man die einfachere Bewegungsgleichung

$$
\frac{\partial \widetilde{\mathscr{V}}_{N}}{\partial t}+\sum_{i=1}^{N} \frac{\bar{\Delta} \overline{\mathfrak{F}}_{N}}{\bar{\Delta} \mathfrak{R}_{v}^{(i)}} \mathfrak{V}_{v}^{(i)}=0,
$$

wobei das Symbol $\bar{\Delta} / \bar{\Delta} \Re_{v}{ }^{(i)}$ besagt, daß alle in erster Ordnung nur langsam veränderlichen Größen konstant zu halten sind. Das Potentialglied ist also herausgemittelt. Diese „grobe“ Gleichung beachtet nicht mehr die Verhältnisse im kleinen und gibt nur noch den Mittelwert von $\widetilde{\mho}_{N}$ in größeren Bereichen an. Andererseits stellt (24) dann tatsächlich einen Energieerhaltungssatz dar.

\section{§ 5. Schluß}

Wir haben damit das gewünschte Ziel erreicht. Die Irreversibilität ist aus der Quantentheorie heraus verständlich. Sie ist in gewissem Sinne sogar ein quantentheoretischer Effekt. Die Quantentheorie

\footnotetext{
8 Vgl. auch die Habilitationsschrift des Verf.; bisher unveröffentlicht.
} 
schreibt nämlich vor, daß man den Gleichungen ihre Genauigkeit nehmen muß und sie sagt auch, wie man es machen muß.

Noch ein Hinweis auf die Ähnlichkeit dieser Ergebnisse mit denen von va n K a m pen ${ }^{4}$. Der Unterschied liegt im wesentlichen darin, daß wir hier nicht nur den in der Natur nie vorliegenden „reinen Fall", sondern beliebige Gemische in Betracht ziehen und uns ferner auf den konkreten Phasenraum beziehen konnten. Dies drückte sich darin aus, daß wir statt der Annahme des irregulären Verhaltens der Phasenwinkel aller in Betracht zu ziehenden Wellenfunktionen (s. van Kampen, §9) physikalisch interpretierbare und darum sehr durchsichtige Annahmen über die einzelnen Wellenfunktionen gemacht haben.
Die Onsager sche irreversible Thermodynamik (s. z. B. de $\operatorname{Groot}{ }^{9}$ ) folgt unmittelbar aus dem statistischen Grundgesetz (17) zusammen mit der Symmetrierelation (18), wie va n $\mathrm{K}$ a m pe ${ }^{4}$ gezeigt hat.

Man kann die hier gewonnene irreversible Statistik wieder etwas verfeinern, indem man auf die hier durchgeführte „Vergröberung“ dann verzichtet, wenn die Koordinaten zweier Teilchen einander nahekommen. Das führt zur Boltzmannschen Stoßgleichung. Hierüber berichtet eine andere Arbeit des Verfassers ${ }^{5}$.

Für die Förderung dieser Arbeit und Diskussion zu den hier angeschnittenen Fragen habe ich Herrn Professor L u d wi g sehr zu danken.

9 S. R. D e Groot, Thermodynamics of Irreversible Processes, North-Holland Publ. Comp., Amsterdam 1951.

\title{
Temperaturabhängigkeit der magnetischen und optischen Eigenschaften organischer Stikstoff-Radikale
}

\author{
Von K. H. Hausser \\ Aus dem Max-Planck-Institut für Medizinische Forschung, Institut für Chemie, Heidelberg \\ (Z. Naturforschg. 11 a, 20-32 [1956]; eingegangen am 18. November 1955)
}

\begin{abstract}
Beim N-Äthyl-phenazyl(I), bei Wursters Blau-Perchlorat(II) und bei verschiedenen TetrazoliumRadikalen ist die paramagnetische Suszeptibilität bei tiefer Temperatur wesentlich kleiner, als nach dem Curieschen Gesetz zu erwarten wäre; in einigen Fällen verschwindet sie fast ganz. Beim N-Äthyl-phenazyl und bei Wursters Blau-Perchlorat treten mit sinkender Temperatur neue, sehr langwellige Absorptionsbanden auf.

Möglichkeiten zur Deutung beider Effekte werden diskutiert. Das Verschwinden des Paramagnetismus und das Auftreten der neuen Absorptionsbande bei tiefen Temperaturen wird auf eine Kopplung der $\pi$-Elektronen-Systeme von scheibenförmig aufeinanderliegenden Molekülen zurückgeführt. Aus der Konzentrations- und Temperaturabhängigkeit der langwelligen Bande folgt, daß zwei Phenazyle bei tiefer Temperatur eine Absorptionseinheit bilden; ihre Dissoziationswärme ergibt sich zu etwa $+2 \mathrm{kcal} / \mathrm{mol}$.
\end{abstract}

Seit Le w is ${ }^{1}$ vor etwa 30 Jahren erkannte, daß freie Radikale auf Grund ihrer ungeraden Elektronenzahl paramagnetisch sein müssen und sich dadurch von allen anderen organischen Verbindungen unterscheiden, wurden zahlreiche Radikale von verschiedenen Autoren, vor allem von $\mathrm{Müller}$ und seinen Mitarbeitern, auf ihre magnetischen Eigenschaften untersucht ${ }^{2}$. Dabei ergab sich, daß alle bisher bekannten organischen freien Radikale in fester, kristallisierter Form angenähert dem Curieschen bzw. dem Curie-Weißschen Gesetz folgen mit relativ kleinen Werten der Korrekturgröße $\Theta$.

1 G. N. L e w i s, Chem. Rev. 1, 231 [1925].

2 E. M ülle r, Angew. Chem. 64, 233 [1952]; dort ausführliche Literaturangaben.
Eigene Messungen aus neuerer Zeit ${ }^{3}$ hatten das Ergebnis, daß bei einigen Radikalsalzen auch in festem Zustand starke Abweichungen vom Curieschen Gesetz auftreten. Das Ziel dieser Arbeit ist,

1. die Ergebnisse von magnetischen Messungen an weiteren Radikalen und Radikalsalzen mit anomaler Temperaturabhängigkeit des Paramagnetismus mitzuteilen,

2. die magnetischen Meßresultate mit den Absorptionsspektren bei Zimmertemperatur und bei der Temperatur des flüssigen Stickstoffs zu vergleichen und

${ }^{3}$ K. H. H a u s s e r u. H. K a in e r, Z. Naturforschg. 9a, 783 [1954]; Chem. Ber. 86, 1563 [1953]. 